\title{
Effect of Nonstructural Protein 2 Hypervariable Regions in the Replication of Porcine Reproductive and Respiratory Syndrome Virus in Marc-145 Cells
}

\author{
Ying Liu ${ }^{\mathrm{a}}$ Feng-Xue Wang ${ }^{\mathrm{a}}$ Yong-Jun Wen ${ }^{\mathrm{a}}$ Zhen-Guang Li ${ }^{\mathrm{a}}$ Xing Liu ${ }^{\mathrm{a}}$ \\ Na Sun ${ }^{a}$ Yong Yang ${ }^{a}$ Shu-Qin Zhang ${ }^{a}$ Hong-Wei Zhu ${ }^{a}$ Shi-Peng Cheng ${ }^{a}$ \\ Hua Wu ${ }^{b}$ \\ a State Key Laboratory for Molecular Biology of Special Economic Animals, Institute of Special Economic Animal

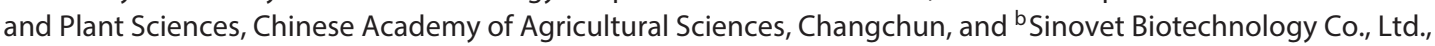 \\ Beijing, China
}

\section{Key Words}

Nonstructural protein $2 \cdot$ Transiently expressed amino acids - Truncated polypeptides - Porcine reproductive and respiratory syndrome virus - Viral replication

\section{Abstract}

Background: Highly pathogenic (HP) porcine reproductive and respiratory syndrome virus (PRRSV) causes prolonged high fever, red discoloration of the body, blue ears and a high mortality. Previously, we found that the PRRSV vaccine strain TJM contained a deletion of 120 amino acids (aa 628747) in nonstructural protein 2 (Nsp2). We aimed to explore the replication features of PRRSV after adding the transiently expressed product of these 120 aa in vitro. Methods: We constructed seven eukaryotic expression plasmids containing different parts of the 120-aa sequence, transfected them into Marc-145 cells and then inoculated the cells with $10^{3}$ $\mathrm{TCID}_{50}$ TJM per well. We detected virus replication at mRNA and protein level by real-time RT-PCR and Western blotting, respectively, and determined the virus titer. Results: The transiently expressed 120 aa and one of its truncated polypeptides inhibited PRRSV TJM propagation on Marc-145 cells. The complete 120-aa sequence induced a remarkable decrease in PRRSV replication, causing a reduction in structural protein levels between 36 and $48 \mathrm{~h}$ after infection. Additionally, aa 628-727 partly reduced the replication of PRRSV on Marc-145 cells. Conclusions: The 120 aa from Nsp2, especially aa 628-727, play a negative role in PRRSV TJM proliferation.

(c) 2015 S. Karger AG, Basel

\section{Introduction}

Porcine reproductive and respiratory syndrome virus (PRRSV), a member of the Arterivirus genus, causes severe reproductive losses, respiratory disease, increased mortality and reduced growth rates in pigs $[1,2]$. It was found primarily in North America and Western Europe during the early 1990s, and has resulted in immense economic losses for the swine industry worldwide $[3,4]$.

PRRSV contains a single-strand positive-sense RNA genome, approximately $15 \mathrm{~kb}$ in length, comprising at least 10 open reading frames (ORF) [5-7]. ORF1a and ORF1b, located in the $5^{\prime}$-proximal three quarters of the

Ying Liu and Feng-Xue Wang contributed equally to this work.

\section{KARGER 125}

(c) 2015 S. Karger AG, Base

$0300-5526 / 15 / 0585-0288 \$ 39.50 / 0$

E-Mail karger@karger.com

www.karger.com/int
Hua $\mathrm{Wu}$

Sinovet (Beijing) Biotechnology Co., Ltd., B302 Zhongguancun Biomedical Park

5 Shangdikaituo Road

Beijing 100085 (China)

E-Mailwuhua@sinovetah.com 
Table 1. Oligonucleotide primers used in this study

\begin{tabular}{|c|c|c|c|}
\hline Primer & Gene location & Sequence & Peptide fragments \\
\hline $\mathrm{P} 0 \mathrm{~F}$ & $3118-3146$ & $5^{\prime}$ AGAGCGCTGATGTCAAGTGTTAAGATCAC 3' AfeI & Nsp2 (628-747) \\
\hline P0R & $3475-3498$ & 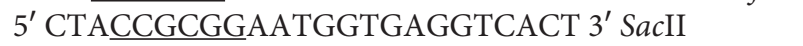 & Nsp2 (628-747) \\
\hline P1R & $3419-3437$ & $5^{\prime}$ ATCCGCGGCCCGCACGGGT $33^{\prime}$ SacII & Nsp2 (628-727) \\
\hline P2R & $3353-3377$ & 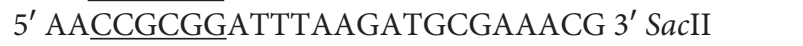 & Nsp2 (628-707) \\
\hline P3R & $3293-3317$ & 5' TACCGCGGATCCCACATGCGAGAGA 3' SacII & Nsp2 (628-687) \\
\hline $\mathrm{P} 1 \mathrm{~F}$ & $3178-3202$ & 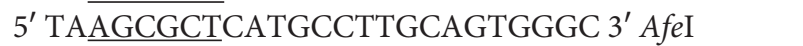 & Nsp2 (648-747) \\
\hline $\mathrm{P} 2 \mathrm{~F}$ & $3238-3262$ & 5' GTAGCGCTCATGTGTGATGCGTCCA 3’ AfeI & Nsp2 (668-747) \\
\hline P3F & $3298-3324$ & $5^{\prime}$ AGAGCGCTCATGAGGGTTGACATGCTG 3'AfeI & Nsp2 (688-747) \\
\hline $\mathrm{N}-\mathrm{F}$ & $14791-14816$ & $5^{\prime}$ CGGGATCCATGCCAAATAACAACGGC $3^{\prime}$ BamHI & $\mathrm{N}$ protein \\
\hline $\mathrm{N}-\mathrm{R}$ & $15155-15180$ & $5^{\prime}$ CTGCGGCCGCTCATGCTGAGGGTGAT $3^{\prime}$ NotI & $\mathrm{N}$ protein \\
\hline
\end{tabular}

viral genome, are translated to produce two large viral replication proteins, polyproteins ppla and pplab, respectively $[8,9]$. These two precursors generate at least 14 nonstructural proteins (Nsp) by autocatalytic processing. ORF1a encodes Nsp1 $\alpha$, Nsp1 $\beta$, Nsp2-Nsp6, Nsp7 $\alpha$, Nsp7 $\beta$ and Nsp8, and ORF1b encodes Nsp9-Nsp12 [8, $10-12]$.

Nsp2, the largest Nsp ( 2.9 kb) is notable for its genetic heterogeneity and relevance to virus replication [13-16]. It is associated with PRRSV cell and tissue tropism, and may participate in multiprotein assembly, forming viral replication complexes [17]. Nsp2 is encoded by $21-23 \%$ of the genome and is predicted to have four domains: an N-terminal cysteine protease domain (PLP2/ OTU), a large hypervariable domain (HV), a transmembrane domain and a relatively conserved carboxyl domain (C-terminal) [18-21]. Natural mutations, insertions and especially deletions have frequently been found in the Nsp2 HV of field strains [17, 22-26].

In 2006, a highly pathogenic (HP) strain of PRRSV that contained a discontinuous deletion of 30 amino acids (aa) within the Nsp2 coding region was isolated from diseased piglets [27]. PRRSV TJM is an attenuated strain that was created by passaging the HP-PRRSV TJ strain on Marc-145 cells in our laboratory [28]. One characteristic difference of the PRRSV TJM strain compared with the TJ strain is an additional 120 -aa deletion in Nsp2 (aa 628747). Our previous work demonstrated that Nsp2 can regulate viral replication by gene silencing and overexpression [15]. Mutations using a programmed -2 ribosomal frameshifting to prevent nsp2TF expression impaired PRRSV replication and produced a small-plaque phenotype [29]. Earlier work showed that PRRSV TJM was better adapted to Marc-145 cells and had higher viral titer than PRRSV TJ [15]. Here, we explored the effect of the transiently expressed Nsp2 aa 628-747 and its truncated polypeptides on the regulation of viral replication and searched for the region responsible for this effect.

\section{Methods}

\section{Cells and Viruses}

Marc-145 cells, an African green monkey kidney epithelial cell line that supports PRRSV replication, were grown in minimum essential medium (MEM) supplemented with $6 \%$ fetal bovine serum (FBS). The cell lines were grown at $37^{\circ} \mathrm{C}$ with $5 \% \mathrm{CO}_{2}$. HP-PRRSV TJ strain (NCBI GenBank; accession No. EU860248) was proliferated in Marc- 145 cells. The PRRSV TJM vaccine strain was attenuated from the HP-PRRSV TJ strain on Marc-145 cells.

\section{Design and Construction of the Expression Cassettes}

Eight polymerase chain reaction (PCR) primers (table 1) were designed to amplify seven segments containing the $360 \mathrm{bp}$ that encode the 120 aa of Nsp2 (aa 628-747) missing in the PRRSV TJM, as well as six truncated segments. Each forward primer contains the specific restriction site $A f e I$ and the reverse primer contains SacII. The viral RNAs were extracted from the culture supernatants of PRRSV-infected Marc-145 cells using TRIzol reagent (Invitrogen, USA). Seven fragments were subsequently amplified by PCR with Pfu polymerase (NEB) according to the manufacturer's protocol. These segments were cloned into pEGFP-N1 with restriction enzymes and sequenced using synthetic oligonucleotides (Invitrogen). Sequence data were edited and analyzed using BioEdit software.

\section{Polyclonal Antibody of Nucleocapsid Protein of PRRSV}

The nucleocapsid (N) gene was amplified from PRRSV TJM strain RNA using the primer pair N-F/R (table 1) and was cloned into pGEX-6p-1 with the specific restriction enzymes BamHI and NotI. The recombinant plasmid pGEX-6p-1-N was transformed into Escherichia coli BL21 to express the N protein. The recombinant prokaryotic pGEX-6p-1-N was expressed and purified. Then, the recombinant protein $\mathrm{N}$ was injected into female Balb/c mice, inducing the manufacture of anti-N polyclonal antibodies for the detection of $\mathrm{N}$ protein in PRRSV-infected cells. 
Transfection of Plasmid and Infection with PRRSV

Marc-145 cells were seeded in 12- or 6-well plates at a density of approximately $5 \times 10^{5}$ cells $/ \mathrm{ml}$ and cultured for $24 \mathrm{~h}$ in MEM with $6 \%$ FBS, reaching approximately $80 \%$ confluency. Plasmids used for transfection were purified with the EndoFree Plasmid Mini Kit (Omega) according to the manufacturer's instructions and were quantified with an ultraviolet spectrophotometer. Transfection with $0.16 \mathrm{mg}$ of each plasmid expressing a deletion segment was performed using Lipofectamine ${ }^{\mathrm{TM}} 2000$ (Invitrogen) according to the manufacturer's protocol. The pEGFP-N1 vector was transfected as a control. Five hours after transfection, the culture medium was replaced with fresh MEM containing 2\% FBS and the cells were challenged with PRRSV TJM strain at a multiplicity of infection (MOI) of 1 at this time. Untransfected cells that were infected with PRRSV TJM strain were used as a control. The transfection efficiency was determined by monitoring the enhanced green fluorescence protein (EGFP) fluorescence intensity of transfected cells on an inverted fluorescent microscope. Samples were collected at 24, 36, 48 and $60 \mathrm{~h}$ post infection (hpi) for further analysis, and the experiment was repeated three times.

\section{Quantitative RT-PCR}

The same volume of samples described above was used for quantitative analysis. Total cell RNA or virus RNA was extracted $24,36,48$ or 60 hpi using TRIzol reagent (Invitrogen). For reverse transcription (RT)-PCR, total extracted RNA (dissolved in $9 \mu \mathrm{ldi}$ ethyl pyrocarbonate-treated water) was mixed with random primers (TaKaRa; $1 \mu \mathrm{l}$ ), dNTP mix (TaKaRa; $2 \mu \mathrm{l}$ ), $40 \mathrm{U}$ rRNasin (Promega; $0.5 \mu \mathrm{l}$ ), and $200 \mathrm{U}$ M-MLV reverse transcriptase (Promega; $1 \mu \mathrm{l})$. These mixtures were incubated at $42^{\circ} \mathrm{C}$ for $1 \mathrm{~h}$ and inactivated at $95^{\circ} \mathrm{C}$ for $10 \mathrm{~min}$.

Quantitative PCR (qPCR) was carried out using GoTaq ${ }^{\circledR}$ Probe qPCR Master Mix (Promega) on an MJ Mini Personal Thermal Cycler (Bio-Rad). Each reaction was performed in a $20-\mu$ l volume [10 $\mu$ l qPCR master mix, $0.5 \mu \mathrm{l}$ specific primers $(10 \mathrm{~mm})$ and $2 \mu \mathrm{l}$ cDNA template]. The cycling parameters were $94^{\circ} \mathrm{C}$ for $2 \mathrm{~min}$, $95^{\circ} \mathrm{C}$ for $30 \mathrm{~s}$, followed by 40 cycles of $95^{\circ} \mathrm{C}$ for $30 \mathrm{~s}, 52.9^{\circ} \mathrm{C}$ for $30 \mathrm{~s}$ and $72^{\circ} \mathrm{C}$ for $10 \mathrm{~s}$. A dissociation stage was included at the end of each reaction as a quality control step. A melt curve analysis was performed at the end of each assay.

\section{Western Blot Analysis}

At $48 \mathrm{hpi}$, the cell lysates were prepared by adding $500 \mathrm{ml} \mathrm{RIPA}$ lysis buffer (100 mM Tris-Base, $\mathrm{pH}$ 8.0; 0.1\% SDS; $1 \%$ Triton $\mathrm{X}-100 ; 1 \%$ deoxycholate and $150 \mathrm{mM} \mathrm{NaCl}$ ). The proteins were separated on an SDS $15 \%$ gradient polyacrylamide gel and transferred to a PVDF membrane (0.22 $\mu \mathrm{m}$; Millipore); the membrane was blocked by incubation in a saturation buffer (phosphate-buffered saline containing $5 \%$ dry milk and $0.05 \%$ Tween 20; PBST) for $1 \mathrm{~h}$. The membrane was reacted with anti-N polyclonal mouse antibodies and with anti- $\beta$-actin (Sigma) monoclonal mouse antibody as internal standard, subsequently incubated at $37^{\circ} \mathrm{C}$ for $1 \mathrm{~h}$, and then washed three times with PBST. The membrane was incubated with horseradish peroxidase-labeled anti-mouse (Sigma) antibodies at $37^{\circ} \mathrm{C}$ for $1 \mathrm{~h}$, and washed three times with PBST. The membrane was treated with chemiluminescent eECL from a Western blot kit (Cwbio) and imaged using chemiluminescent film or digital capture.

\section{Viral Titration}

The titers of virus proliferating on Marc-145 cells from all experimental groups were determined. Marc-145 cells were seeded into 96-well plates 1 day before infection. Supernatants were collected, serially diluted 10 -fold using MEM with $2 \%$ FBS, and added to the plates at $100 \mu \mathrm{l} /$ well in eight equal sets. Five days after infection, the virus titers were determined using a microtitration infectivity assay and the titers were recorded as a $50 \%$ tissue culture infective dose $\left(\mathrm{TCID}_{50}\right)$ per milliliter using the Reed-Muench method.

\section{Results}

\section{Generation of Recombinant Plasmid}

The plasmid designs were based on the sequences of the whole Nsp2 120-aa segment (aa 628-747) and the inner six segments, which differ from one another by 20 aa and partially overlap with one another (fig. 1). PCR results confirmed that the target genes were successfully cloned into the pEGFP-N1 vector (Invitrogen). Nsp2 (628-747) was $360 \mathrm{bp}$ (fig. 2). The sizes of the other six segments were $300 \mathrm{bp}$ [Nsp2 (628-727)], 240 bp [Nsp2 (628-707)], 180 bp [Nsp2 (628-707)], 300 bp [Nsp2 (648-747)], $240 \mathrm{bp}$ [Nsp2 (668-747)] and $180 \mathrm{bp}$ [Nsp2 (688-747)] (fig. 2).

\section{Identification of Target Gene Expression}

Marc-145 cells were transfected with the recombinant plasmid, and cell monolayers were harvested at the time points indicated in the Methods. The transfection efficiency was determined by monitoring the EGFP fluorescence intensity of the transfected cells on an inverted fluorescent microscope. At 36 hpi, EGFP fluorescence was detected in all of the transfected plasmids. To determine the transfection efficiency, we merged fluorescent and visible light images (fig. 3). The results showed that the efficiency of transfection was up to $30 \%$.

\section{Effects of Nsp2 (628-747) and Truncated Polypeptides} on Viral RNA Synthesis and on the Regulation of PRRSV TJM Replication in Marc-145 Cells

To investigate if the transiently expressed products of these segments have effects on viral replication, we performed qRT-PCR to measure the levels of viral RNA copy numbers 24, 36 and 48 hpi. Our results show that the transfected segments had a regulatory effect on PRRSV TJM replication (fig. 4). Nsp2 (628-747) had a strong inhibitory effect during the early stages of infection (24-48 hpi). Nsp2 (628-727) inhibited replication $(\mathrm{p}<0.05)$ only between 36 and 48 hpi. We collected samples at $60 \mathrm{hpi}$, 
Fig. 1. a Fragmentation strategy for the Nsp2 region that is present in the HPPRRSV TJM strain but is missing in the PRRSV TJ strain. These segments differ from one another by 20 aa and partially overlap. The position range is denoted below the reference alignment to the TJ Nsp2 protein sequence. $\mathbf{b}$ The aa sequences used were shown. Each peptide was attained by amplification, cloning and sequencing.

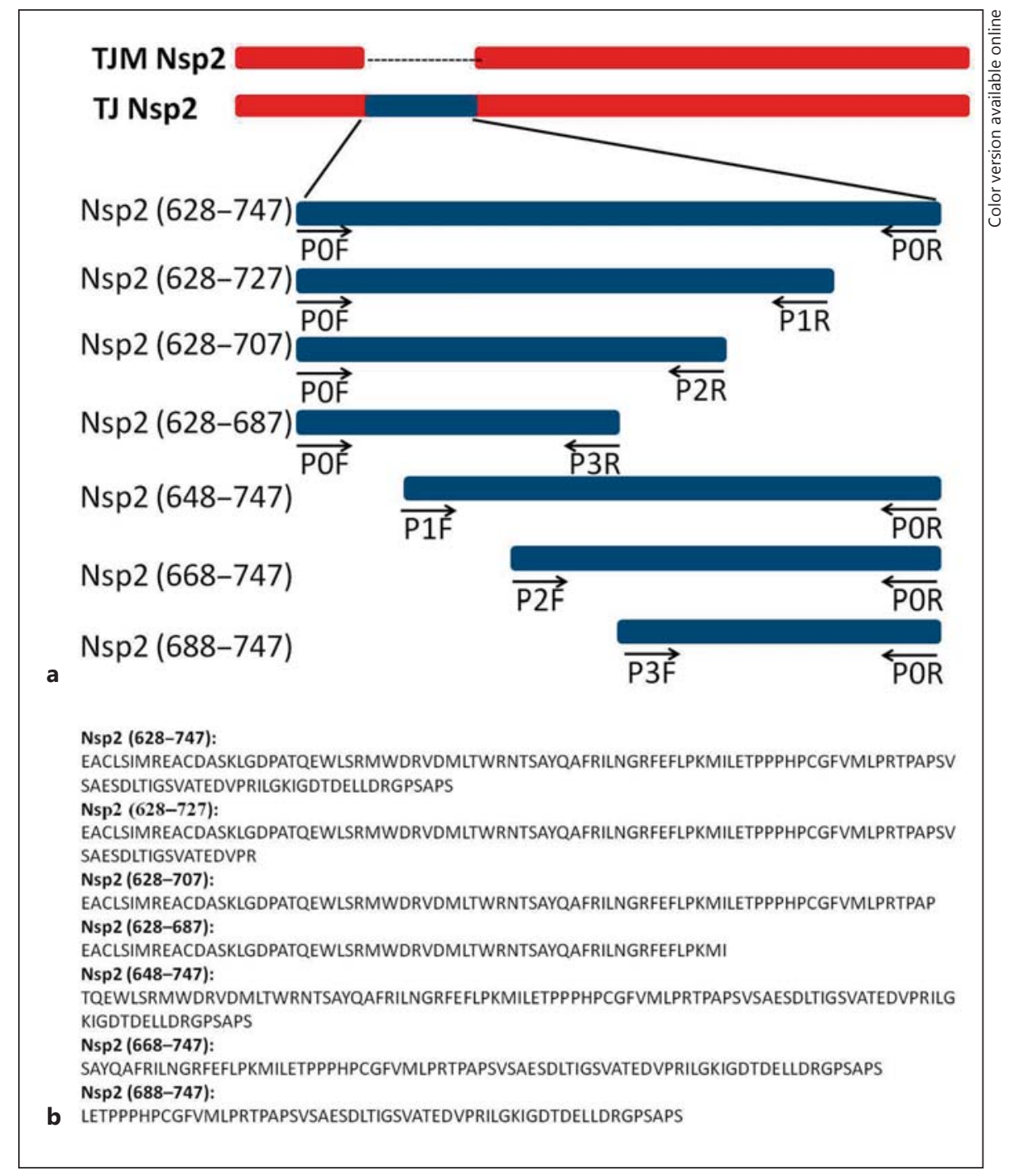

but there were no significant differences between the viral RNA copy numbers of any of the groups at this time. None of the other truncated polypeptides had an effect on viral replication that was significantly different from that of the control treatment.

\section{Viral Protein Synthesis in Transfected Cells}

We were able to detect the inhibitory effect of these polypeptides on viral replication not only at gene but also at protein level. The expression level of the $\mathrm{N}$ protein, which is one of the structural proteins, can reflect the quantity of the viral replication. A Western blot analysis was employed on cells at $48 \mathrm{hpi}$ to investigate the levels of $\mathrm{N}$ expression (fig. 5), and $\beta$-actin expression was tested as a control. In agreement with the gene level results, the in- tegrated Nsp2 (628-747) also had the strongest inhibitory effect on $\mathrm{N}$ expression, and Nsp2 (628-727) was the second most capable of suppressing viral replication.

Series of Truncated Polypeptides Suppressed Marc-145 Cell PRRSV Titers

We transfected groups of Marc-145 cells with each expression plasmid and then inoculated the cells with the PRRSV TJM strain at an MOI of 1 and cultured them for $48 \mathrm{~h}$. We used pEGFP-N1 as a control plasmid. The viral titers in the experimental groups transfected with Nsp2 (628-727), Nsp2 (628-707) or Nsp2 (628-747) were all lower than the viral titer of the control group (fig. 6). The viral titer of cells transfected with the complete 120 -aa segment, Nsp2 (628-747), was $10^{6.875} \mathrm{TCID}_{50}$, which was 


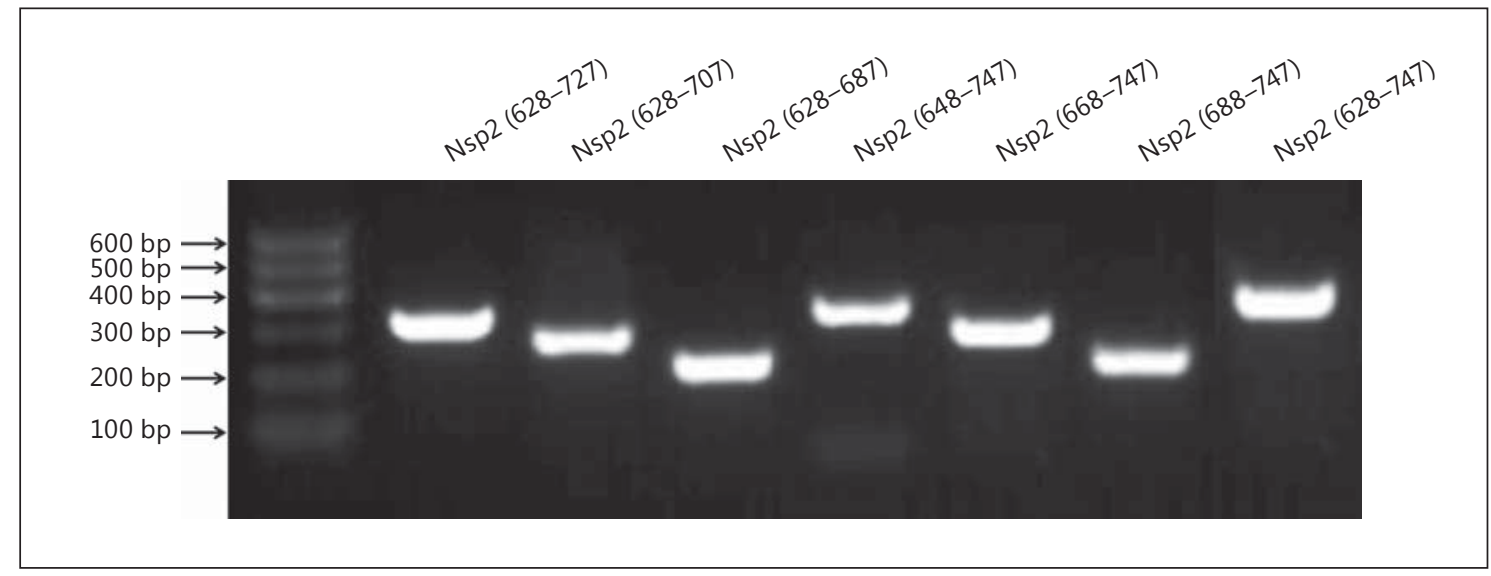

Fig. 2. PCR of the recombinant plasmids containing the series of deletion segments. Seven pairs of primers (table 1) amplifying different segments of the Nsp2 deletion region which are the whole Nsp2 (628-747) segment and the other six segments from the Nsp2 120-aa deletion segment. They were used to confirm the creation of recombinant plasmids.
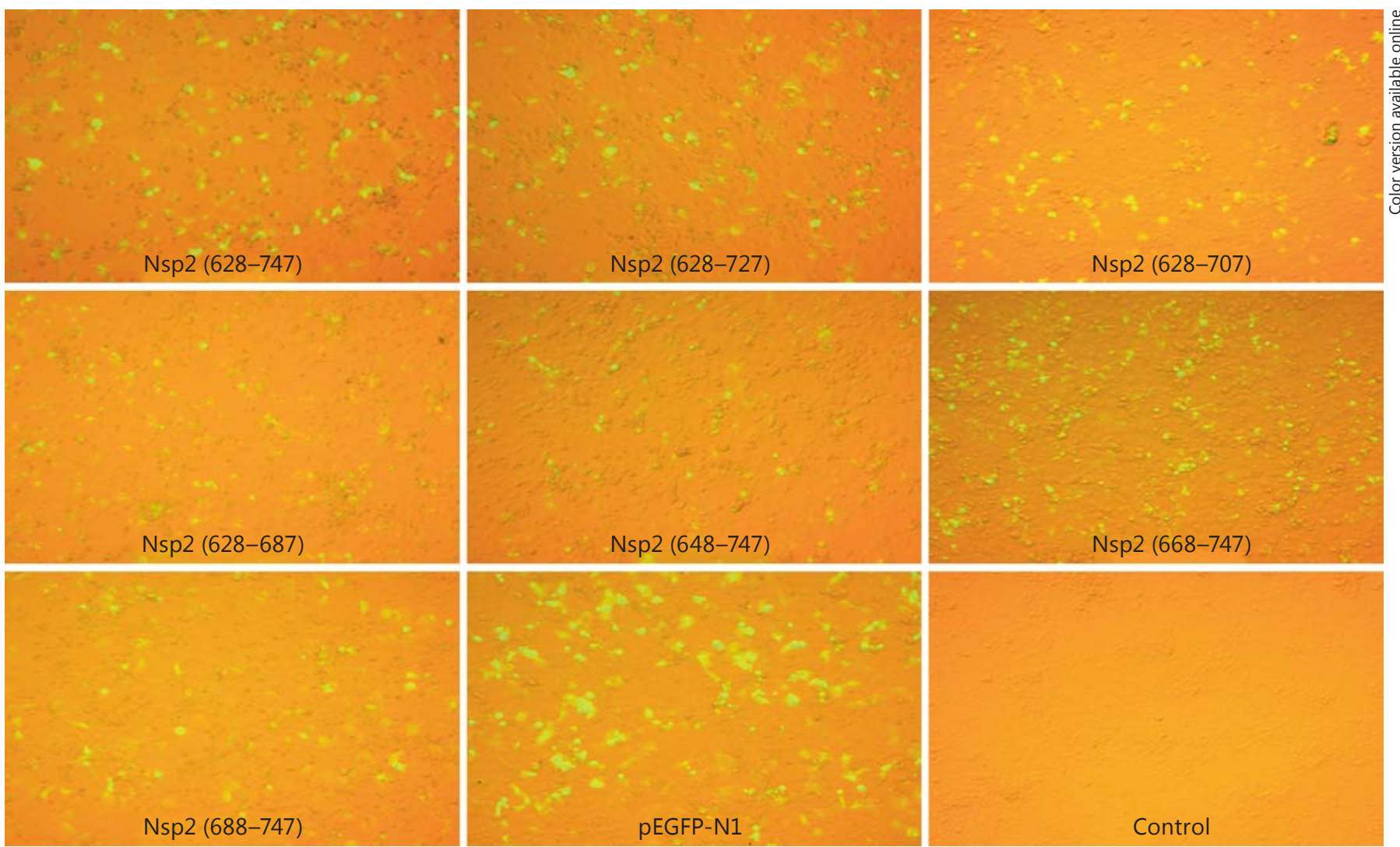

Fig. 3. Transfection efficiencies of the series of recombinant plasmids in Marc-145 cells. The EGFP fluorescence of different recombinant plasmids $36 \mathrm{~h}$ after transfection. pEGFP-N1 and no treatment were used as the positive and negative controls, respectively. 

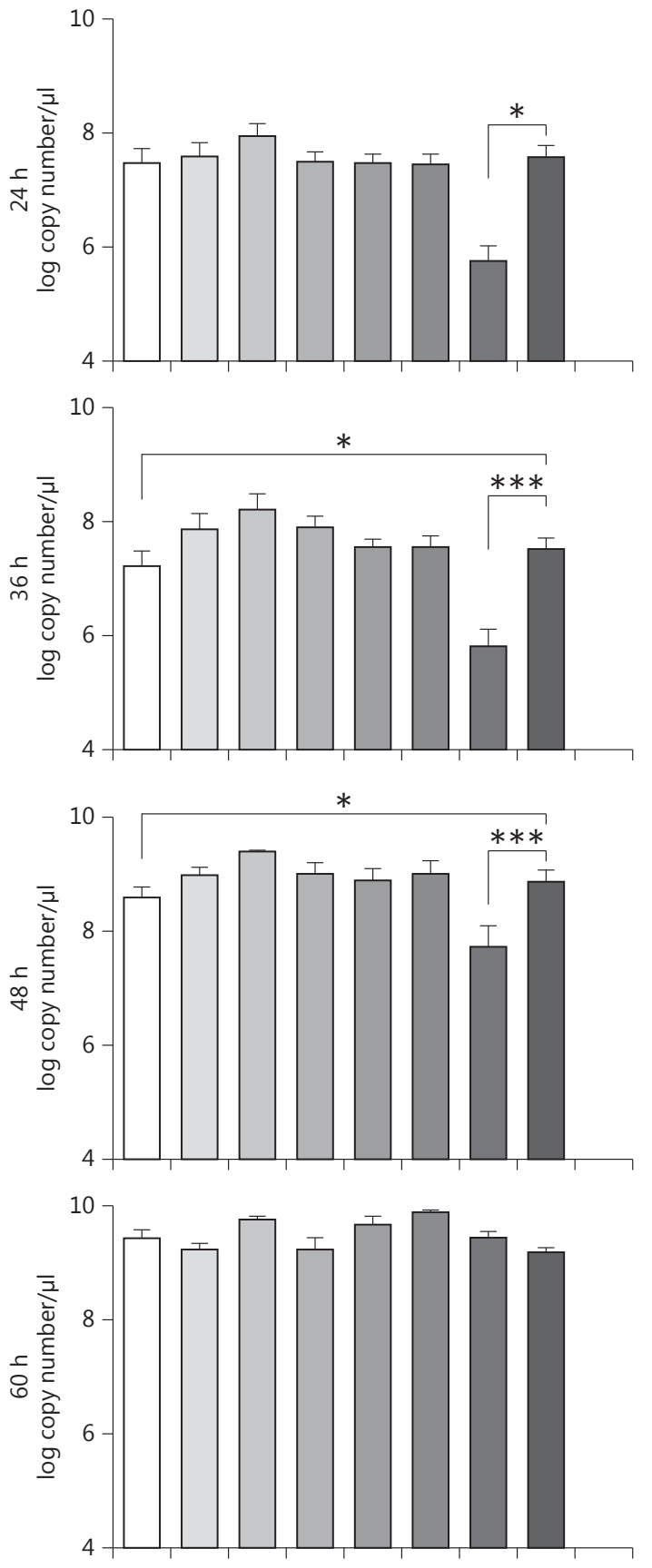

Nsp2 (628-727)

Nsp2 (628-707)

Nsp2 (628-687)

Nsp2 (648-747)

Nsp2 (668-747)

Nsp2 (688-747)

Nsp2 (628-747)

pEGFP-N1

TJM significantly lower than the viral titer of the control group $\left(10^{7.375} \mathrm{TCID}_{50}\right)$. The $\mathrm{TCID}_{50}$ of the cells transfected with the other Nsp2 segments ranged from $10^{7.125}$ to $10^{7.500}$. The TCID 50 of cells transfected with these segments was close to that of the cells transfected with the pEGFP-N1 control, and they were not significantly different from each other. The cytopathic effects of cells in the experimental groups transfected with Nsp2 (628-727, 628-707 or 628-747) were smaller and difficult to see under a microscope. Additionally, the abilities of each segment to inhibit the viral titer as measured by $\mathrm{TCID}_{50}$ were in agreement with the corresponding copy numbers detected by qRT-PCR, which measures the gene level, and with the corresponding amount of proteins detected by Western blotting, which measures the protein level, for each segment.

\section{Discussion}

The effectiveness of viral replication contributes to the virulence of the virus and its ability to successfully infect the host. Arterivirus Nsp2 can collaborate with Nsp3 to form the double-membrane vesicles derived from paired endoplasmic reticulum membranes, which provide the sites for viral RNA synthesis [30, 31]. Nsp2 is also considered to be one of the crucial viral proteins in PRRSV replication, and it is believed to participate in multiprotein assembly during the formation of viral replication complexes [17]. One study showed that PRRSV replication in host cells prevents the fusion between autophagosomes and lysosomes, which causes an accumulation of autophagosomes that may serve as a viral replication site. Nsp2 colocalizes with microtubule-associated protein 1 light chain kinase (LC3), which can activate the autophagy machinery [32]. Additionally, Nsp2 can be detected as a structural component in purified PRRSV virions, which suggests that Nsp2 is relevant to viral replication [33].

Each section of Nsp2 plays a different role in PRRSV replication. The PLP2 region possesses both trans- and cis-cleavage activities involved in viral replicase polyprotein processing and is cleaved at the Nsp2-Nsp3 (Nsp2/3)

Fig. 4. Copy numbers of viral RNA detected by qRT-PCR. Marc145 cells were transfected with recombinant plasmids and challenged with the PRRSV TJM strain. Culture supernatants were harvested 24, 36, 48 and 60 hpi. Total RNA was isolated and qRTPCR was performed to determine viral RNA copy numbers. Data are shown as means $\pm \mathrm{SD}, \mathrm{n}=3 .^{*} \mathrm{p}<0.05,{ }^{* * *} \mathrm{p}<0.01$. 


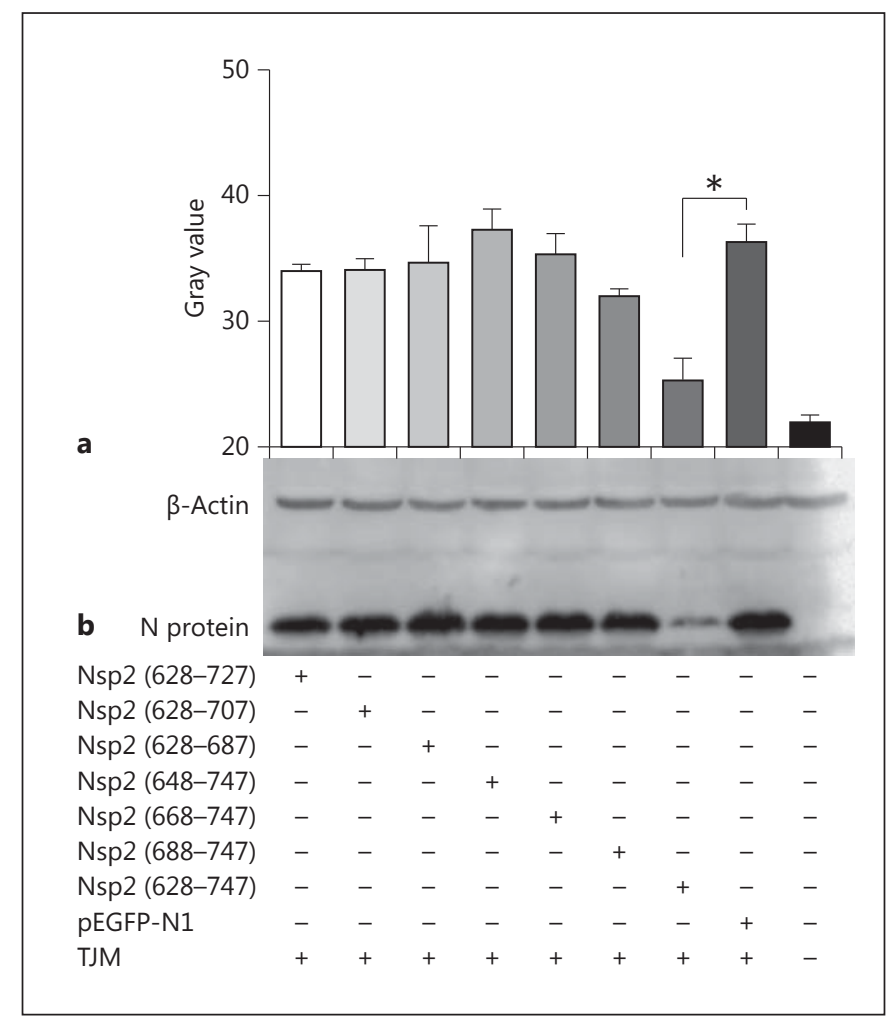

Fig. 5. $\mathrm{N}$ protein expression after transfection with the series of Nsp2 deletion region segments at $48 \mathrm{hpi}$. Western blotting of proteins in Marc-145 cells infected with the PRRSV TJM strain at an MOI of 1 was performed to test the expression levels of $\mathrm{N}$ protein. Cell lysates were fractionated by SDS-PAGE, and after transfer, the membranes were reacted with mouse antibodies specific for $\mathrm{N}$, as indicated. Anti- $\beta$-actin antibodies were used as a loading control. Western blotting was repeated at least three times. The gray value is the average value evaluated by BandScan 5.0. a The gray value of western blotting band. $\mathbf{b}$ The result of western blotting for detecting $\mathrm{N}$ protein of PRRSV TJM. ${ }^{*} \mathrm{p}<0.05$.

junction $[18,34]$. Additionally, although the Nsp2 PLP2 domain (aa 13-35) is dispensable for viral replication, the core domain (aa 47-180) and the immediate downstream region (aa 181-323) are essential for viral replication [35]. The Nsp2 C-terminal region is not related to viral replication because it can tolerate an insertion of EGFP, and the resulting product of the progeny virus is not affected [36]. The Nsp2 HV region has a complicated role in viral replication. Some studies suggest that a deletion in part of the Nsp2 HV region inhibits viral replication $[37,38]$. In contrast, some deletions in this region make PRRSV replication more efficient $[39,40]$. Additionally, some fragments of the HV region were found to be nonessential for PRRSV [41, 42]. Previous research deduced that aa 47-

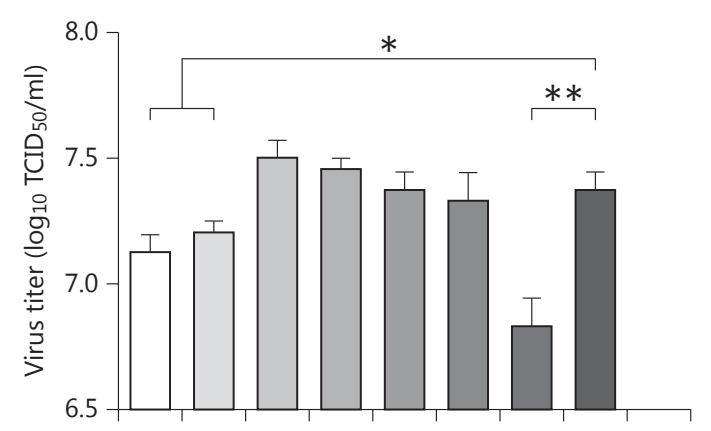

Nsp2 (628-727)

Nsp2 (628-707)

Nsp2 (628-687)

Nsp2 (648-747)

Nsp2 (668-747)

Nsp2 (688-747)

Nsp2 (628-747)

pEGFP-N1

TJM

$\begin{array}{ccccccccc}+ & - & - & - & - & - & - & - & - \\ - & + & - & - & - & - & - & - & - \\ - & - & + & - & - & - & - & - & - \\ - & - & - & + & - & - & - & - & - \\ - & - & - & - & + & - & - & - & - \\ - & - & - & - & - & + & - & - & - \\ - & - & - & - & - & - & + & - & - \\ - & - & - & - & - & - & - & + & - \\ + & + & + & + & + & + & + & + & -\end{array}$

Fig. 6. Virus titers of the PRRSV TJM strain in the transfected cells at 48 hpi. Cells were infected at an MOI of 1 with the PRRSV TJM strain. Samples were collected at $48 \mathrm{hpi}$. The results shown are mean values from three replications of the experiment, and viral titers are expressed as $\mathrm{TCID}_{50}$ per milliliter. ${ }^{*} \mathrm{p}<0.05,{ }^{* *} \mathrm{p}<0.01$.

323, 627-727 and 758-813 are all important for PRRSV replication [43].

The diversity of the Nsp2 gene is attributable to functional changes. Deletions may lead to abated restrictions, similarly to the effects of Nsp deletions in other plague viruses [44]. In our previous study, we found that the viral load was significantly lower in the sera from pigs infected with the PRRSV TJM stain, which has a 120-aa deletion in the HV region of Nsp2, than in those infected with the HP-PRRSV TJ strain [28]. However, the PRRSV TJM strain has a higher virus titer in Marc-145 cells in vitro than the HP-PRRSV TJ strain. The results from Nsp2 gene interference tests demonstrated that inhibition of Nsp2 expression significantly reduced the replication of the HP-PRRSV TJ strain in Marc- 145 cells. Following infection with the HP-PRRSV TJ strain, viral titers were higher on the two Marc-145 cell lines that were constructed to express TJ-Nsp2 and TJM-Nsp2 than they were in unaltered Marc-145 cells, which suggests that overex- 
pressed Nsp2 plays a positive role in PRRSV proliferation [15]. Recently, the presence of Nsp2 associated with viral particles of diverse PRRSV strains was verified [33], which reveals that Nsp2 exists in or on viral particles as multiple isoforms, including a HP one.

Here, we focus on Nsp2, especially the 120-aa segment deleted in PRRSV TJM. Our aim was to investigate the effect of the 120-aa segment and its truncated polypeptides on PRRSV TJM replication. Our results show that the complete 120-aa segment has a significant influence on PRRSV TJM replication. It inhibited the replication of PRRSV during the early stages of infection (24- $48 \mathrm{hpi}$ ), and its effect was observed at both gene and protein level. Two shorter truncated polypeptides, Nsp2 (628-727 and 628-707), were identified as having an inhibitory effect at subsequent time points (36 and $48 \mathrm{hpi}$, respectively). They suppressed the viral replication more than any other segment except for the $\Delta \mathrm{Nsp} 2$ (complete 120 -aa segment). Therefore, we hypothesize that there is an acute fragment in the head of the 120-aa segment. The epitope aa sequence, TRPKYSAQAIIDSG, was located at aa 633647 in Nsp2 and was shown to exist in or on viral particles [33]. Here, we confirmed that $60-80$ aa of the 120 aa may play a leading role and 1-20 aa of Nsp2 (628-747) may play a secondary role in inhibiting virus replication. This suggests that the 60-80 aa of Nsp2 (628-747) may contain another epitope, but further investigation is required to test this idea.

\section{Conclusions}

The 120 aa from Nsp2 hypervariable regions, especially aa 628-727, play a negative role in PRRSV TJM proliferation.

\section{Acknowledgments}

The study was financially supported by grants from the National 'Twelfth Five-Year' High Technology Research and Development Program (2011AA10A213), the Agricultural Science and Technology Innovation Program (CAAS-ASTIP-2014-ISAPS) and the Key Technology R\&D Program of Jilin Province (20140204073NY).

\section{Disclosure Statement}

The authors declare that they have no competing interests.

\section{References}

1 Ellingson JS: Porcine Reproductive and Respiratory Syndrome Virus: Diagnostic Update and Search for Novel Modified Live Vaccines; thesis, Iowa State University, 2013, http://lib.dr.iastate.edu/etd/13006.

2 Guo B, Lager KM, Henningson JN, et al: Experimental infection of United States swine with a Chinese highly pathogenic strain of porcine reproductive and respiratory syndrome virus. Virology 2013;435:372-384.

3 Neumann EJ, Kliebenstein JB, Johnson CD, et al: Assessment of the economic impact of porcine reproductive and respiratory syndrome on swine production in the United States. J Am Vet Med Assoc 2005;227:385-392.

4 Pejsak Z, Markowska-Daniel I: Losses due to porcine reproductive and respiratory syndrome in a large swine farm. Comp Immunol Microbiol Infect Dis 1997;20:345-352.

5 Firth AE, Zevenhoven-Dobbe JC, Wills NM, et al: Discovery of a small arterivirus gene that overlaps the GP5 coding sequence and is important for virus production. J Gen Virol 2011;92:1097-1106.

6 Oh J, Lee C: Proteomic characterization of a novel structural protein ORF5a of porcine reproductive and respiratory syndrome virus. Virus Res 2012;169:255-263.
7 Stadejek T, Stankevicius A, Storgaard T, et al: Identification of radically different variants of porcine reproductive and respiratory syndrome virus in Eastern Europe: towards a common ancestor for European and American viruses. J Gen Virol 2002;83:1861-1873.

8 Snijder EJ, Meulenberg JJ: The molecular biology of arteriviruses. J Gen Virol 1998;79: 961-979.

9 Yun SI, Lee YM: Overview: replication of porcine reproductive and respiratory syndrome virus. J Microbiol 2013;51:711-723.

10 Chen Z, Lawson S, Sun Z, et al: Identification of two auto-cleavage products of nonstructural protein 1 (nsp1) in porcine reproductive and respiratory syndrome virus infected cells: nspl function as interferon antagonist. Virology 2010;398:87-97.

11 Fang Y, Snijder EJ: The PRRSV replicase: exploring the multifunctionality of an intriguing set of nonstructural proteins. Virus Res 2010;154:61-76.

12 Li Y, Tas A, Snijder EJ, Fang Y: Identification of porcine reproductive and respiratory syndrome virus ORF1a-encoded non-structural proteins in virus-infected cells. J Gen Virol 2012;93:829-839.
13 Yoshii M, Okinaga T, Miyazaki A, et al: Genetic polymorphism of the nsp2 gene in North American-typ porcine reproductive and respiratory syndrome virus. Arch Virol 2008;153:1323-1334.

14 Liu JK, Wei CH, Yang XY, et al: Genetic diversity and evolutionary characterization of Chinese porcine reproductive and respiratory syndrome viruses based on NSP2 and ORF5. Arch Virol 2013;158:1811-1816.

15 Wang FX, Wen YJ, Yang BC, et al: Role of non-structural protein 2 in the regulation of the replication of the porcine reproductive and respiratory syndrome virus in MARC145 cells: effect of gene silencing and over expression. Vet Microbiol 2012;161:58-65.

16 Choi HW, Nam E, Lee YJ, et al: Genomic analysis and pathogenic characteristics of type 2 porcine reproductive and respiratory syndrome virus nsp2 deletion strains isolated in Korea. Vet Microbiol 2014;170:232245.

17 Fang Y, Kim DY, Ropp S, et al: Heterogeneity in Nsp2 of European-like porcine reproductive and respiratory syndrome viruses isolated in the United States. Virus Res 2004;100:229235. 
18 Han J, Rutherford MS, Faaberg KS: The porcine reproductive and respiratory syndrome virus nsp2 cysteine protease domain possesses both trans- and cis-cleavage activities. J Virol 2009;83:9449-9463.

19 Sun Z, Chen Z, Lawson SR, Fang Y: The cysteine protease domain of porcine reproductive and respiratory syndrome virus nonstructural protein 2 possesses deubiquitinating and interferon antagonism functions. J Virol 2010;84:7832-7846.

20 Sun Y, Han M, Kim C, et al: Interplay between interferon-mediated innate immunity and porcine reproductive and respiratory syndrome virus. Viruses 2012;4:424-446.

21 Chen Z, Zhou X, Lunney JK, et al: Immunodominant epitopes in nsp2 of porcine reproductive and respiratory syndrome virus are dispensable for replication, but play an important role in modulation of the host immune response. J Gen Virol 2010;91:10471057.

22 Allende R, Lewis TL, Lu Z, et al: North American and European porcine reproductive and respiratory syndrome viruses differ in nonstructural protein coding regions. J Gen Virol 1999;80:307-315.

23 Han J, Wang Y, Faaberg KS: Complete genome analysis of RFLP 184 isolates of porcine reproductive and respiratory syndrome virus. Virus Res 2006;122:175-182.

24 Shen S, Kwang J, Liu W, Liu DX: Determination of the complete nucleotide sequence of a vaccine strain of porcine reproductive and respiratory syndrome virus and identification of the Nsp2 gene with a unique insertion. Arch Virol 2000;145:871-883.

25 Ropp SL, Wees CE, Fang Y, et al: Characterization of emerging European-like porcine reproductive and respiratory syndrome virus isolates in the United States. J Virol 2004;78: 3684-3703.

26 Zhou L, Yang X, Tian Y, et al: Genetic diversity analysis of genotype 2 porcine reproductive and respiratory syndrome viruses emerging in recent years in China. Biomed Res Int 2014;2014:748068.
27 Tian K, et al: Emergence of fatal PRRSV variants: unparalleled outbreaks of atypical PRRS in China and molecular dissection of the unique hallmark. PLoS One 2007;2:e526.

28 Leng X, Yu X, Zhao T, et al: Mutations in the genome of the highly pathogenic porcine reproductive and respiratory syndrome virus potentially related to attenuation. Vet Microbiol 2012;157:50-60.

29 Fang Y, Treffers EE, Li Y, et al: Efficient -2 frameshifting by mammalian ribosomes to synthesize an additional arterivirus protein. Proc Natl Acad Sci USA 2012;109:E2920E2928.

30 Knoops K, Bárcena M, Limpens RW, et al: Ultrastructural characterization of arterivirus replication structures: reshaping the endoplasmic reticulum to accommodate viral RNA synthesis. J Virol 2012;86:2474-2487.

31 Snijder EJ, van Tol H, Roos N, Pedersen KW: Non-structural proteins 2 and 3 interact to modify host cell membranes during the formation of the arterivirus replication complex. J Gen Virol 2001;82:985-994.

32 Sun MX, Huang L, Wang R, et al: Porcine reproductive and respiratory syndrome virus induces autophagy to promote virus replication. Autophagy 2012;8:1434-1447.

33 Kappes MA, Miller CL, Faaberg KS: Highly divergent strains of porcine reproductive and respiratory syndrome virus incorporate multiple isoforms of nonstructural protein 2 into virions. J Virol 2013;87:13456-13465.

34 Han J, Rutherford MS, Faaberg KS: Proteolytic products of the porcine reproductive and respiratory syndrome virus nsp2 replicase protein. J Virol 2010;84:10102-10112.

35 Han J, Liu G, Wang Y, Faaberg KS: Identification of nonessential regions of the nsp2 replicase protein of porcine reproductive and respiratory syndrome virus strain VR-2332 for replication in cell culture. J Virol 2007;81: 9878-9890.

36 Kim DY, Calvert JG, Chang KO, et al: Expression and stability of foreign tags inserted into nsp2 of porcine reproductive and respiratory syndrome virus (PRRSV). Virus Res 2007; 128:106-114.
37 Gauger PC, Faaberg KS, Guo B, et al: Genetic and phenotypic characterization of a 2006 United States porcine reproductive and respiratory virus isolate associated with high morbidity and mortality in the field. Virus Res 2012;163:98-107.

38 Faaberg KS, Kehrli ME Jr, Lager KM, Guo B, Han J: In vivo growth of porcine reproductive and respiratory syndrome virus engineered nsp2 deletion mutants. Virus Res 2010;154: 77-85.

39 Ran ZG, Chen XY, Guo X, et al: Recovery of viable porcine reproductive and respiratory syndrome virus from an infectious clone containing a partial deletion within the Nsp2-encoding region. Arch Virol 2008;153:899-907.

$40 \mathrm{Ni}$ YY, Huang YW, Cao D, et al: Establishment of a DNA-launched infectious clone for a highly pneumovirulent strain of type 2 porcine reproductive and respiratory syndrome virus: identification and in vitro and in vivo characterization of a large spontaneous deletion in the nsp2 region. Virus Res 2011;160: 264-273.

41 Xu YZ, Zhou YJ, Zhang SR, et al: Stable expression of foreign gene in nonessential region of nonstructural protein 2 (nsp2) of porcine reproductive and respiratory syndrome virus: applications for marker vaccine design. Vet Microbiol 2012;159:1-10.

42 Guo B, Vorwald AC, Alt DP, et al: Large scale parallel pyrosequencing technology: PRRSV strain VR-2332 nsp2 deletion mutant stability in swine. Virus Res 2011;161:162-169.

43 Wang FX, Song N, Chen LZ, et al: Non-structural protein 2 of the porcine reproductive and respiratory syndrome (PRRS) virus: a crucial protein in viral pathogenesis, immunity and diagnosis. Res Vet Sci 2013;95:1-7.

44 Moser C, Stettler P, Tratschin JD, Hofmann MA: Cytopathogenic and noncytopathogenic RNA replicons of classical swine fever virus. J Virol 1999;73:7787-7794. 